Dysomnias were related to comorbid oppositional defiant disorder and stimulant medication, but not to ADHD. Parasomnias were similar in clinical and nonclinical controls. Sleep-related involuntary movements were increased in frequency in children with the combined subtype of ADHD and in those with anxiety. Although children with $\mathrm{ADHD}$ had more sleep problems than normal children, they did not differ from those in the control group referred for a clinical assessment. (Corkum P, Moldofsky H, Hogg-Johnson S, Humphries T, Tannock R. Sleep problems in children with attention-deficit/hyperactivity disorder: Impact of subtype, comorbidity, and stimulant medication. I Am Acad Child Adolesc Psychiatry October 1999;38:1285-1293). (Respond: Dr Tannock, Department of Psychiatry Research, Hospital for Sick Children, 555 University Avenue, Toronto, Ontario, Canada M5G 1X8).

COMMENT. Children with ADHD, in comparison with normal children, experience more sleep problems evidenced by dyssomnias and involuntary movements, but the frequency of parasomnias is similar to that in normally developing peers. Except for the association of sleep-related involuntary movements and the combined subtvpe of ADHD, sleep problems in ADHD children do not differ from those in clinical comparison groups without ADHD. Dysomnias in children with ADHD occur especially in those with comorbid ODD or in children taking stimulant medication. Involuntary movements during sleep are strongly associated with anxiety, especially separation anxiety.

The International Classification of Children's Sleep Disorders, a more comprehensive and current listing than the DSM, includes 1) dyssomnias, or primary sleep disorders (eg. obstructive sleep apnea, narcolepsy, periodic limb movements, inappropriate environment and parenting, circadian sleep-wake rhythm disorders); 2) parasomnias (eg. sleepwalking and sleep terrors - arousal from deep NREM sleep, headbanging - sleep-wake transition disorders, nightmares - with REM sleep; and 3) sleep disorders associated with neuropsychiatric or other medical disorders (eg. depression, epilepsy, asthma). (Stores G. Children's sleep disorders: modern approaches, developmental effects, and children at special risk. Dev Med Child Neurol Aug 1999;41:568-573). The effects of sleep disorders on cognition, emotional state and behavior are discussed, and the need for a greater professional awareness of the problem is stressed.

\title{
SLEEP DISORDERS IN BLIND CHILDREN
}

The frequency and type of sleep disorders in 77 blind children, from 3 to 18 years of age, were compared with 79 matched controls, using a 42-item questionnaire in a study at the Stanford University Sleep Disorders Center, Stanford, CA, and the Laboratoire du Sommeil, Centre Hospitalier Universitaire de l'Hotel-Dieu a Paris, France. Questions were derived from the DSM-IV and the ICSD90, and were answered by the child alone or with parental help. None had mental retardation nor any chronic illness aside from blindness. Only $8 \%$ had a circadian rhythm disorder (dyschronosis). Blindness occurred in perinatal life in $75 \%$ and after 1 year of life in $25 \%$.

Mean total sleep time was similar in both groups; it was $526+/-61$ minutes on weekdays and $600 \mathrm{~min}$ on weekends. A significantly greater proportion of blind children (17\%) slept less than 7 hours per night on weekdays compared to controls (2.6\%), and blind subjects had more sleep complaints (difficulty in getting to sleep, awakening too early, poor quality sleep, sleepwalking, bruxism). Daily episodic involuntary sleepiness occurred in 13\% of blind children compared to $1.3 \%$ of controls. Age of onset of blindness and presence or absence of light 
perception had no influence on the complaints of insomnia. (Leger D, Prevot E, Philip P et al. Sleep disorders in children with blindness. Ann Neurol Oct 1999;46:648-651). (Respond: Dr C Guilleminault, Stanford University Sleep Disorder Center, 401 Quarry Road, Suite 3301, Stanford, CA 94305).

COMMENT. Insomnia is more common in blind children than controls, with early awakening particularly on school days. The resultant daytime sleepiness may have an adverse effect on learning.

Melatonin treatment of insomnia in children is reviewed from the University of British Columbia, Vancouver, Canada (Jan JE, Freeman RD, Fast DK. Dev Med Child Neurol Aug 1999;41:491-500). The light-dark cycle is the strongest "zeitgeber,"an entraining factor that adjusts the function of the suprachiasmatic nucleus of the anterior hypothalamus and the endogenous circadian brain rhythms by environmental stimuli. Circadian disturbances can result from psychiatric and neurologic disorders, environmental factors, and use of drugs which interfere with pineal melatonin (MLT) secretion. Lack of appreciation of environmental zeitgebers may occur in mentally retarded or blind children, although the incidence was low in the Stanford study. Chronic disabilities may alter the perception of cues for synchronizing sleep with the environment, leading to sleep-wake cycle disorders. Abnormal endogenous MLT secretion has been reported in blind children with sleep disorders, especially those with cortical visual impairment, and in patients with cerebral palsy, or brain tumors involving the hypothalamus, optic chiasma, pineal, or prefrontal cortex. Patients with ocular visual loss are less affected.

The pineal produces MLT in the evening, reaching a peak at $3 \mathrm{am}$. Newborns have no MLT until 3 months, levels increase in the first year and remain stable until early puberty, when they begin to decline. MLT for the treatment of sleep-wake cycle disorders in children is discussed at length in this review article. Fast-release MLT is effective for about 5 hours; time-release MLT may last up to 9 hours. MLT should not be taken at the same time as other drugs or vitamins. Anticonvulsants and food alter the absorption of MLT. The dose recommended by researchers is dependent on age and the cause of the sleep disorder. Low doses $(0.3$ to $0.5 \mathrm{mg})$ are sometimes successful, but larger amounts are usually required: 1 to $3 \mathrm{mg}$ in toddlers and 2.5 to $5 \mathrm{mg}$ for older children. Sleep induction occurs within half an hour. Environmental changes to strengthen zeitgebers and foster healthy sleep habits are required to reset the circadian rhythm. The MLT assists in learning better sleep habits, and cognitive functioning and behavior improve. Once the desired effect is achieved, the MLT may be withdrawn, usually after some months, but depending on the underlying cause of the sleep disorder. Although no serious side effects are reported, the indiscriminate use of MLT is discouraged.

\section{INFECTIOUS DISORDERS}

\section{NEUROLOGIC COMPLICATIONS OF ENTEROVIRUS 71 INFECTION}

The neurologic complications associated with the 1998 Taiwan enterovirus 71 epidemic are reported from National Cheng Kung University, Tainan; Chang Gung Children's Hospital, Kaohsiung; and National Defense Medical Center, Taipei, Taiwan. In 41 children with acute neurological manifestations, the mean age was 2.5 years (range, 3 months to 8 years), 28 (68\%) had hand-foot-and-mouth disease, $6(15 \%)$ had herpangina, and skin or mucosal lesions were absent in 7 . Three neurologic syndromes identified were aseptic meningitis (3 patients $\{7 \%\})$, acute 